M. Simkó*, M. Nentwich, A. Gazsó, U. Fiedeler

\section{Wie kommen Nanopartikel in den menschlichen Körper und was verursachen sie dort?}

\section{Zusammenfassung}

Durch die vielfältigen Anwendungsmöglichkeiten von Nanomaterialien kommen die Menschen auf unterschiedliche Weise mit ihnen in Kontakt. Daher ist es wichtig zu analysieren, inwiefern Nanopartikel in den menschlichen Körper eindringen und welche gesundheitlichen Auswirkungen sie dort verursachen können. Derzeit verdichten sich die Hinweise darauf, dass die toxische Wirkung von Nanopartikeln umso größer ist, desto kleiner sie sind. Neben der Größe sind auch die Form und die chemische Beschaffenheit der Nanomaterialien für ihre biologische Wirkung von Bedeutung. Es ist bekannt, dass Nanopartikel Entzündungsreaktionen in der Lunge verursachen können und vereinzelt wurde auch über Lungenfibrosen berichtet. Es gibt Hinweise darauf, dass Nanopartikel in Gefäßwände eindringen und somit bestimmte Dysfunktionen hervorrufen bzw. das Herz-Kreislaufsystem beeinflussen können. Eine neue Studie zeigt sogar, dass nadelförmige, asbestfaserähnliche Nanoröhrchen im Tiermodell chronische Entzündungen auslösen können. Nur wenige Daten gibt es über die Wirkungen im Verdauungstrakt und im Nervensystem sowie über die Aufnahme von Nanopartikeln über die Haut ins Blut. Daher werden hier die möglichen Aufnahmewege von Nanopartikeln in den menschlichen Körper vorgestellt und die wichtigsten Daten diskutiert.

\section{Einleitung}

Die Nanotechnologie und die mit ihr verbundenen Nanomaterialien werden im Bereich der Forschung aber auch schon in vielen auf dem Markt erhältlichen Gebrauchsgütern eingesetzt. Das heißt, dass die allgemeine Bevölkerung bereits mit Nanomaterialien in Kontakt kommt. Es stellt sich daher die Frage, ob die verwendeten $\mathrm{Na}$ nomaterialien in den menschlichen Körper eindringen und ob sie dort gesundheitliche Schäden auslösen können. Die möglichen gesundheitlichen Risiken von Nanopartikeln sind erst wenig erforscht. In verschiedenen Untersuchungen wurde gezeigt, dass ungebundene Nanopartikel aufgrund ihrer geringen Größe mit der Atemluft bis in die feinsten Strukturen der Lunge vordringen, dort Entzündungsreaktionen verursachen und weiter ins Blut gelangen können. Über den Blutkreislauf können sich Nanopartikel im Körper verteilen und in andere Organe eindringen. Es wurde weiterhin gezeigt, dass bestimmte Nanopartikel von Zellen aktiv oder passiv aufgenommen werden, wobei eine schädigende Wirkung nicht ausgeschlossen werden kann. Dabei ist ihre biologische Wirkung nicht nur von der chemischen Zusammensetzung, sondern auch von Größe, Form, Oberflächenbeschaffenheit, Aggregationszustand und Oberflächenladung des Nanopartikels abhängig. Das vorliegende Dossier beschäftigt sich mit den möglichen Eintrittspforten von Nanopartikeln in den menschlichen Körper und beschreibt einige biologische Effekte, die Nanopartikel im Körper hervorrufen.

\section{Eintrittspforten in den menschlichen Körper}

Nanomaterialien können direkł über die Körperöffnungen in den Körper aufgenommen werden, so z. B. durch Einatmen oder durch Verschlucken. Darüber hinaus wird auch diskutiert, dass sie indirekt durch die Hautporen aufgenommen werden können. Die menschliche Haut, der Magen-DarmTrakt und die Lungen sind immer in direktem Kontakt mit der Umwelt. Während die Haut als eine Barriere dient, erlauben der Magen-Darm-Trakt und die Lunge auch den Transport (passiv und/oder aktiv) der verschiedenen Substanzen wie Wasser, Nährstoffe oder Saverstoff. Es erscheint wahrscheinlich, dass die Aufnahme der Nanomaterialien in den menschlichen Körper über diese Wege erfolgt. Aufgrund ihrer geringen Größe sind Nanopartikel in der Lage, die Zellmembran zu durchdringen und so auf subzellulärer Ebene zu agieren. Wie dies passiert, wird gegenwärtig erforscht.

\section{Die Haut}

Die menschliche Haut ist eine echte Barriere zur Umwelt (ausgenommen die Sonnenstrahlung, die für die Vitamin-D-Produktion notwendig ist), über die keine essentiellen Elemente aufgenommen werden.

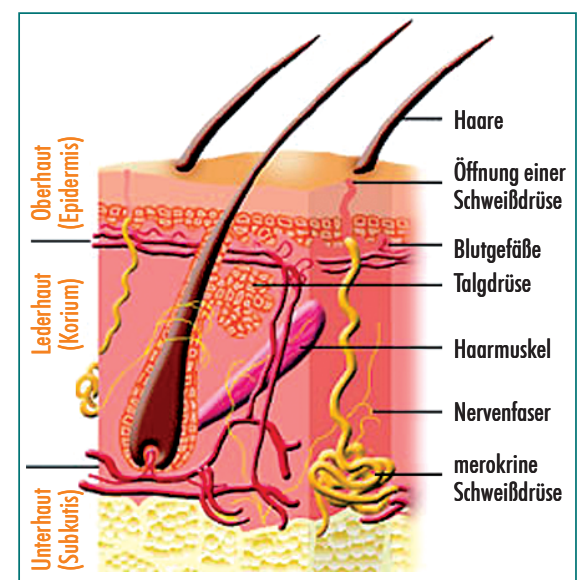


Die Fläche der menschlichen Haut beträg† im Durchschnitt 1,5-2 $\mathrm{m}^{2}$, wobei die erste Schicht der Haut aus einer relativ dicken Schicht toter Zellen (so genannte keratinisierte Zellschicht, $10 \mu \mathrm{m}$ ) besteht (Abb. 1) ${ }^{1}$.

Nanopartikel aus Titan- oder Zinkoxiden werden bereits in großem Umfang in unterschiedlichen kosmetischen Pflegeprodukten, wie etwa in Sonnencremes, als sehr effektiver UV-Absorber eingesetzt. Die Frage stellt sich, ob diese Produkte bzw. die Nanomaterialien die oberen Hautschichten überwinden und sie so in das tiefer gelegene Gewebe gelangen können. Bisher wurde dies nicht eindeutig nachgewiesen. Es gibt jedoch Hinweise, dass sich Teilchen zumindest in den Hauttaschen von Haarwurzeln, den so genannten Haarfollikeln, anreichern können $^{2}$. Da während des Haarwachstums die Haarfollikel geöffnet sind, könnten die Nanopartikel in die tiefere Schichten eindringen. Untersuchungen haben gezeigt, dass die Bestandteile einmassierter partikelfreier Lotion nach sieben Tagen wieder vollständig aus dem Haarfollikel entfernt war, während sich die Zahl der Teilchen aus der partikelhaltigen Lotion nur halbiert hatte ${ }^{3 ; 4}$. Eine Aufnahme ins Blut (Translotion) über die gesunde Haut wurde bislang nicht nachgewiesen.

Im Gegensatz dazu wurde in Untersuchungen mit gesunder Haut festgestellt, dass verschiedene so genannte Quantum Dots in die Haut eindringen können ${ }^{5}$. (Quantum Dots werden aus Halbleitermaterial hergestellt und haben die Eigenschaft, Licht bestimmter Wellenlänge zu absorbieren. Sie finden Verwendung als Biomarker in den Biowissenschaften oder in LEDs und Displays).

Ob eine Partikelaufnahme durch die verletzte oder durch die erkrankte Haut (Schuppenflechte etc.) anderes verläuft, ist Gegenstand der laufenden Untersuchungen. Man ist sich jedoch sicher, dass die Barrierefunktion nicht mehr gewährleistet ist und so das Eindringen von Nanopartikel möglich ist ${ }^{6}$.

\section{Der Verdauungstrakt}

Der gesamte Magen-Darm-Trakt ist im engen Kontakt mit allen oral aufgenommen Materialien. Hier werden alle nötigen Nährstoffe für den Körper (ausgenommen Gase) aufgenommen. Die gesamte Fläche des Magen-Darm-Traktes ist eine komplexe Barriere - doch es ist die wichtigste Pforte für Makromoleküle, die in den Körper gelangen müssen. Vom Magen können nur kleine Moleküle durch das Epithel diffundieren. Das Epithel des Darmes ist in engem Kontakt mit dem bereits vorverdauten Material. So kön-

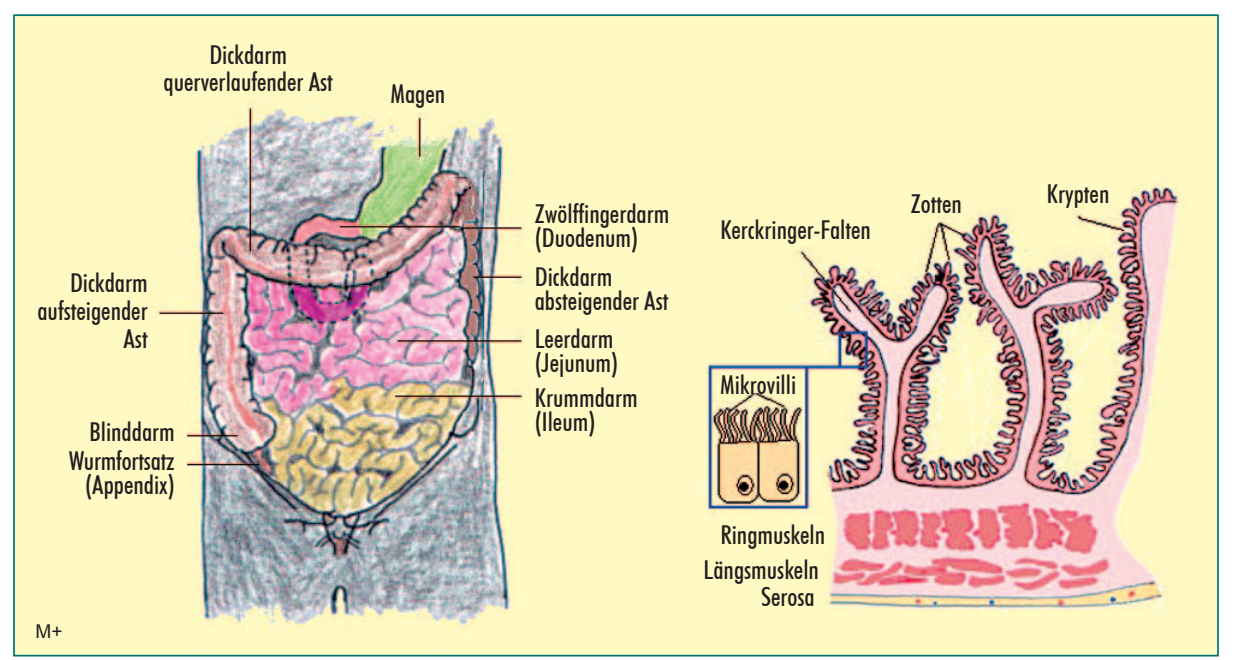

Abbildung 2: Aufbau des menschlichen Magen-Darm-Traktes (links Gesamtübersicht, rechts vergrößerte Ansicht der Darmschleimhaut und der Mikrovilli) ${ }^{7}$

nen Nährstoffe direkt aufgenommen werden. Die Nahrung im Dünndarm ist bereits verdaut und besteht aus einer Mischung von Molekülen wie Disacchariden, Peptiden, Fettsäuren und Monoglyzeriden. Diese werden in den Darmzotten umgewandelt und dann resorbiert (Abb. 2) ${ }^{7}$. Um die Oberfläche des Epithels zu vergrößern sind die Darmzotten selbst mit kleineren Zotten (Microvilli) bedeckt. So entsteht eine Fläche von ca. $200 \mathrm{~m}^{2}$ für die Aufnahme von Nährstoffen im Magen-Darm-Trakt.

Nanoskalige Strukturen können durch Nahrungsmittel, aber auch nach dem Einatmen über den mukoziliären Rücktransport aus den Bronchien unbewusst verschluckt werden und so in den Magen-Darm-Trakt gelangen. Es wird geschätzt, dass eine Person täglich durchschnittlich $10^{12}$ bis $10^{14}$ Nanound Mikropartikel oral aufnimmt ${ }^{8}$, wobei es sich größtenteils um Silikate und Titandioxid handelt. So passieren 50 bis $100 \mathrm{~nm}$ große Polystyrolpartikel im Tierversuch die Darmwand und gelangen ins Lymphsystem ${ }^{9}$, wogegen Fullerene eher nicht aufgenommen werden. Andere Studien zeigen hingegen keine Aufnahme in das Blutgefäßsystem über dem Magen-Darm-Trakt ${ }^{10,11}$. Offensichtlich besteht kein Konsens darüber, wie sich $\mathrm{Na}$ nopartikel im Magen-Darm-Trakt verhalten. Eine Studie untersuchte die Aufnahme von radioaktiv markierten, intravenös verabreichten Fullerenen im Vergleich zur Aufnahme durch den Magen-Darm-Trakt von Ratten. Das oral aufgenommene Material wurde zu $98 \%$ ausgeschieden, während das intravenös verabreichte Material nach einer Woche zu ca. $80 \%$ in der Leber deponiert war ${ }^{12}$. Dies könnte ein Hinweis darauf geben, dass die Aufnahme von Nanopartikeln über den Darm eher von geringer Bedeutung ist. Nur wenige Studien haben sich bisher mit der Aufnahme und dem Verbleib von Nanopartikeln im Magen-Darm-Trakt beschäftigt, daher kann noch keine abschließende Bewertung erfolgen.

\section{Die Lunge}

Die Lunge besteht aus zwei unterschiedlichen Funktionsbereichen, nämlich aus den Atemwegen, wo die Luft in die oder aus der Lunge transportiert wird, und dem Gasaustauschbereich (Bronchien, Bronchiolen, Alveolen), wo Sauerstoff und Kohlendioxyd mit der Umwelt ausgetauscht wird. Die menschliche Lunge besteht etwa aus 2.300 Kilometern Atemwegen und ca. 300 Million Lungenbläschen (Alveolen) (Abb. 3) ${ }^{13}$. Die Fläche der menschlichen Lunge beträgt etwa $140 \mathrm{~m}^{2}$ und bietet somit eine gewaltige Expositionsfläche. Die Atemwege fungieren wie eine verhältnismäßig robuste Barriere aus einer aktiven Epithelschicht, welche durch eine zähflüssige Schleimschicht (Mucus) geschütz† wird (Luft-Blut-Gewebeschranke). Im Gasaustauschbereich ist die Barriere zwischen der alveolaren Wand und den Kapillaren sehr dünn. Die Luft im Inneren (Lumen) der Lungenbläschen ist nur einige $\mathrm{Na}$ nometer entfernt vom fließenden Blut. In Tierversuchen wurde gezeigt, dass Nanopartikel in der Lage sind, diese Luft-Blut-Gewebeschranke zu überwinden. Somit können Nanomaterialien in den Blutkreislauf des Körpers transportiert werden ${ }^{14}$. Durch die große Oberfläche der Alveolen und den intensiven Luft-Blut-Kontakt sind die Alveolen Umwelteinflüssen gegenüber mehr ausgesetzt als die Atemwege. Die Bronchien sind mit einer Flimmerzellschicht (mukoziliären Schicht) ausgekleidet, um abgelagerte Partikel aus der Lunge zu entfernen (mukoziliäre Clearance). Mit Hilfe der mukoziliä- 


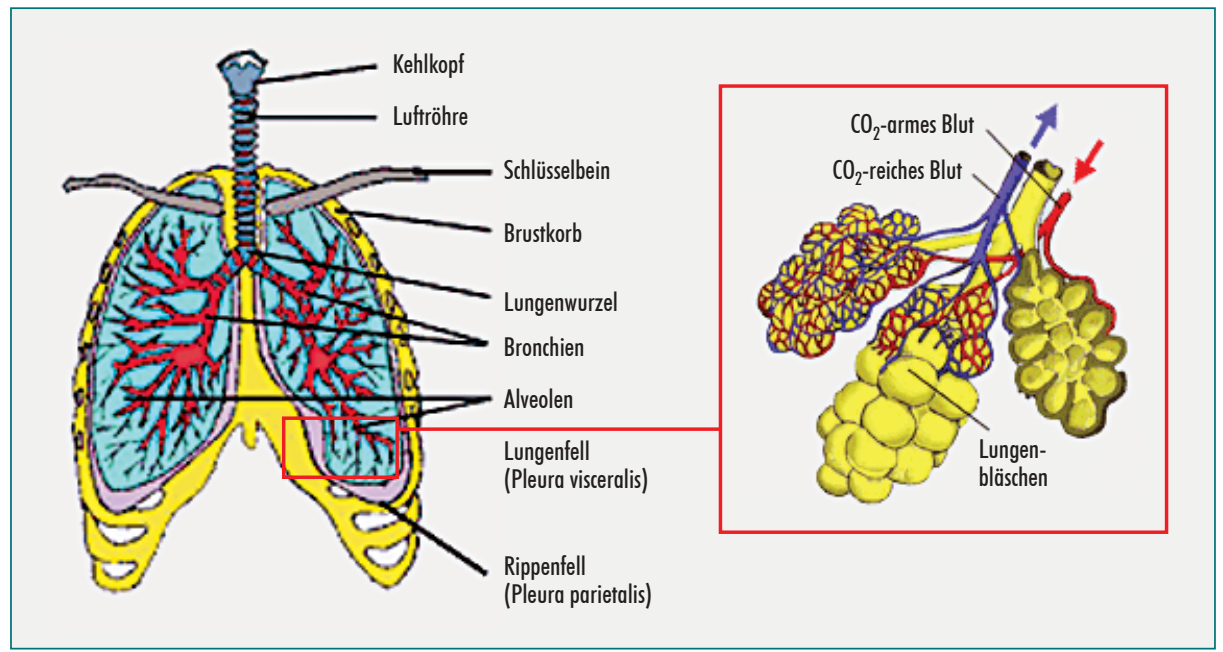

Abbildung 3: Aufbau der menschlichen Lunge (links Gesamtübersicht, rechts vergrößerte Ansicht der Lungenbläschen ${ }^{13}$

ren Clearance werden die meisten entlang der Bronchien abgelagerten Nanopartikel beseitigt. Dieser Mechanismus ist umso ineffektiver, je kleiner die Partikel sind. Das bedeutet, dass Nanopartikel in die Lungenbläschen eindringen, sich auf das Epithel absetzen und danach eine direkte Wechselwirkung mit den Lungenbläschenepithelien stattfinden kann. Da in den Alveolen kein mukoziliärer Clearancemechanismus vorhanden ist, übernehmen so genannte Fresszellen (Makrophagen) die Fremdkörperentfernung.

Auch hier spielt die geringe Größe der Partikel eine Schlüsselrolle. Dass die üblichen Reinigungsmechanismen versagen können, belegt unter anderem die Beobachtung, dass unlösliche Nanopartikel in Tierversuchen über Monate bis Jahre in Bronchien und Alveolen deponiert werden ${ }^{15}$. Höhere Ablagerungsraten sind bei Patienten mit chronischer Bronchitis und Asthma bronchiale beobachtet worden, wobei die verminderte Clearance und die erhöhte Atemfrequenz bei diesen Patienten als Ursache diskutiert wird $^{15,16}$.

Wie lange die aufgenommenen Nanopartikel im Körper verweilen (Kinetik) ist heute noch unklar. Manche Untersuchungen deuten darauf hin, dass u. a. nach dem Einatmen der Nanopartikel diese in die Organe übertreten können. Es wurde gezeigt, dass eingeatmete Nanopartikel nach 7 Tagen in der Leber, Milz, Gehirn, Nieren, Herz und Knochenmark wiederzufinden sind ${ }^{12,17}$. Eine Verstoffwechslung (Metabolisierung) aufgenommener anorganischer Nanopartikel (z. B. Titandioxid) erscheint eher unwahrscheinlich, während organische Nanomaterialien (z. B. Fullerene) eher durch metabolische Prozesse verändert werden können.
Ob die Partikel in den Lymph-/Blutkreislauf übertreten und sich im Körper verteilen und eventuell anreichern und ob sie das HerzKreislauf-System beeinflussen, ist Gegenstand laufender Untersuchungen.

Ebenso wird erforscht, ob während des Atmens aufgenommene z. B. über den Riechnerv ins Gehirn gelangte Partikel im Gehirn verbleiben und sich eventuell dort anreichern können ${ }^{18,19}$. Es wurde nachgewiesen, dass Nanopartikel über diesen Weg auch zu den höheren Gehirnzentren vordringen können (Cortex, Thalamus und Kleinhirn), wobei das Elektroenzephalogramm ein verändertes Muster zeigte.

\section{Auswirkungen im menschlichen Körper}

Tierversuche belegen, dass Entzündungsreaktionen in den Bronchien und Alveolen durch Kohlenstoff-, Polystyrol-, Eisen-, Titandioxid- und Iridiumpartikel in Nanometergröße ausgelöst werden können ${ }^{17,20}$. In Einzelfällen wurden Entzündungsreaktionen bei beruflich exponierten Personen mit nanoskalierten Indium-Zink-Oxiden ${ }^{21}$ und Zirkonium Partikeln aus Schweißrauch beschrieben $^{22}$.

Es gibt einige Daten über die Wirkungen von Nanopartikel, wobei ein enger Zusammenhang zwischen Oberflächenbeschaffenheit und biologischer Wirkung besteht. So wurde z. B. gezeigt, dass direkt in die Lunge verabreichtes Titandioxid (bzw. Nickel- und Vanadiumdioxidpartikel) mit einer Größe von $20 \mathrm{~nm}$ bei Ratten und Mäusen mehr Entzündungsreaktionen verursacht als $250 \mathrm{~nm}$ gro-
Be Partikel. Diese und andere Befunde zeigen, dass die Oberfläche wichtiger für die Toxizität ist als die Masse ${ }^{12}$. Weiterhin sind neben der Größe der Oberfläche auch die Oberflächeneigenschaften (z. B. Existenz reaktiver Gruppen an der Oberfläche) ausschlaggebend für die Toxizität. Eine neve Pilotstudie zeigt an einem speziellen Mausmodell, dass intraperitoneal (d. h. über den vom Bauchfell überzogenen Bereich im Körper) verabreichte, lange (ca. $20 \mu \mathrm{m}$ ), nadelförmige Nanoröhrchen chronische Entzündung hervorrufen, während kurze und/oder gekrümmte Nanoröhrchen keine solche Effekte induzieren. Da diese aufgrund ihrer Beschaffenheit (Form, Länge und unlöslich) ähnlich wie Asbestfasern sind, wurde ein vergleichbarer Wirkmechanismus diskutiert ${ }^{23}$.

Von erheblichem Interesse ist die Frage nach einer möglichen krebserzeugenden Wirkung durch eingeatmeten Nanopartikeln. Es wurde gezeigt, dass die Verabreichung hoher Dosen granulärer und biobeständiger $\mathrm{Na}$ nostäube (inertes Bulk-Material) an Ratten, mit einer erhöhten Tumorhäufigkeit verbunden war ${ }^{24}$. Es ist jedoch nicht klar, ob es sich dabei um einen direkten gentoxischen Effekt der Nanopartikel handelt oder ob es sich um sekundäre Folgen handelt, wie die Freisetzung freier Radikale, was bei chronischen Entzündungen der Fall ist. Wie sich geringe Mengen Nanopartikel beim Menschen verhalten und ob sie Krebs induzieren können, kann noch nicht beantwortet werden. Unklarheit herrscht auch darüber, was mit den aufgenommenen Partikeln passiert. Manche Partikel werden von Epithelzellen aufgenommen (internalisiert), wie es u. a. für nanoskaliertes Titandioxid, Gold, Polystyrol und Zirkonium) beschrieben wurde 22,25,26. Das zugrundeliegende Erklärungsmodell ist, dass die freigesetzten freien Radikale möglicherweise den so genannten oxidativen Stress auslösen und die Zellen irreparabel schädigen (Zytotoxizität). Andere Autoren diskutieren die mögliche Anlagerung der Nanopartikel direkt an die DNA, was zu einer gentoxischen Wirkung führen kann ${ }^{20}$. Aufgenommene Nanopartikel können aus der Lunge ins Blut übertreten (translozieren) 10,14,27,28, wobei diese Ergebnisse kontrovers diskutiert werden. Fest steht, dass die Oberflächenbeschaffenheit wie Ladungsverhalten oder Beschichtung der Partikel neben der Größe die biologische Effektivität beeinflussen können ${ }^{12,15}$. 


\section{Fazit}

Die in die Umwelt gelangten Nanopartikel, wie z. B. Partikel aus Kohlenstoff oder Metalloxide mit einem Durchmesser bis zu 100 Nanometer, können biologische Wirkungen auslösen, da sie in Zellen eindringen und deutlich reaktionsfreudiger als größere Partikel sind. Gleichzeitig verbessern sie Kosmetika, ermöglichen neuartige Beschichtungen (z. B. kratzfeste Autolacke) und gelten als Kandidaten für neuartige und viel versprechende medizinische Verfahren und Therapien. Um das mit der sich ausweitenden Verbreitung von $\mathrm{Na}-$ nopartikeln einhergehende Risiko effektiv abschätzen zu können, bedarf es unbedingt weiterer Forschung. Besonders wichtig erscheint dabei die Untersuchung der tatsächlichen Exposition verschiedener Personengruppen, wie z. B. Konsumentlnnen, beruflich exponierten Personen und PatientInnen sowohl bei der Herstellung als auch bei der Anwendung der Nanopartikeln. Außerdem sind die Wirkmechanismen der aufgenommenen Materialien zu erforschen, um ihre Wirkung besser abschätzen zu können.

\section{Anmerkungen und Literaturhinweise}

1 www.qualimedic.de/Haut.html.

2 Lademann, J., Weigmann, H., Rickmeyer, C., Barthelmes, H., Schaefer, H., Mueller, G. und Sterry, W., 1999, Penetration of titanium dioxide microparticles in a sunscreen formulation into the horny layer and the follicular orifice, Skin Pharmacol Appl Skin Physiol 12(5), 247-56.

3 Lademann, J., Richter, H., Schaefer, U. F., Blume-Peytavi, U., Teichmann, A., Otberg, N. und Sterry, W., 2006, Hair follicles - a long-term reservoir for drug delivery, Skin Pharmacol Physiol 19(4), 232-6.

${ }^{4}$ Lademann, J., Schaefer, H., Otberg, N., Teichmann, A., Blume-Peytavi, U. und Sterry, W., 2004, Penetration of microparticles into human skin, Hautarzt 55 (12), $1117-9$.

5 Ryman-Rasmussen, J. P., Riviere, J. E. und Monteiro-Riviere, N. A., 2006, Penetration of intact skin by quantum dots with diverse physicochemical properties, Toxicol Sci 91(1), 159-65.

${ }^{6}$ Rouse, J. G., Yang, J., Ryman-Rasmussen, J. P., Barron, A. R. und Monteiro-Riviere, N. A., 2007, Effects of mechanical flexion on the penetration of fullerene amino acid-derivatized peptide nanoparticles through skin, Nano Lett 7(1), 155-60.

7 www.vitanet.de/media/img/1060267272542 67493/lungenkrebs_lungenaufbau.gif.

8 Lomer, M. C., Thompson, R. P. und Powell, J. J., 2002, Fine and ultrafine particles of the diet: influence on the mucosal immune response and association with Crohn's disease, Proc Nutr Soc 61(1), 123-30.

9 Volkheimer, G., 1974, Passage of particles through the wall of the gastrointestinal tract, Environ Health Perspect 9, 215-25.

10 Kreyling, W. G., Semmler, M., Erbe, F., Mayer, P., Takenaka, S., Schulz, H., Oberdorster, G. und Ziesenis, A., 2002, Translocation of ultrafine insoluble iridium particles from lung epithelium to extrapulmonary organs is size dependent but very low, J Toxicol Environ Health A 65(20), 1513-30.

11 Kanapilly, G. M. und Diel, J. H., 1980, Ultrafine 239PuO2 aerosol generation, characterization and short-term inhalation study in the rat, Health Phys 39(3), 505-19.

12 Oberdorster, G., Oberdorster, E. und Oberdorster, J., 2005, Nanotoxicology: an emer823-39.

13 www.medizinfo.de/gastro/images/ duendarm.jpg. $411-4$. 785-96.

\section{IMPRESSUM:} www.oeaw.ac.at/ita gestellt: epub.oeaw.ac.at/ita/nanotrust-dossiers/ ISSN: 1998-7293 ging discipline evolving from studies of ultrafine particles, Environ Health Perspect 113(7),

14 Nemmar, A., Hoet, P. H., Vanquickenborne, B., Dinsdale, D., Thomeer, M., Hoylaerts, M. F., Vanbilloen, H., Mortelmans, L. und Nemery, B., 2002, Passage of inhaled particles into the blood circulation in humans, Circulation 105(4),

15 Borm, P. J., Robbins, D., Haubold, S., Kuhlbusch, T., Fissan, H., Donaldson, K., Schins, R., Stone, V., Kreyling, W., Lademann, J., Krutmann, J., Warheit, D. und Oberdorster, E., 2006, The potential risks of nanomaterials: a review carried out for ECETOC, Part Fibre, Toxicol 3, 11.

16 Frampton, M. W., Utell, M. J., Zareba, W., Oberdorster, G., Cox, C., Huang, L. S., Morrow, P. E., Lee, F. E., Chalupa, D., Frasier, L. M., Speers, D. M. und Stewart, J., 2004, Effects of exposure to ultrafine carbon particles in healthy subjects and subjects with asthma, Res Rep Health Eff Inst (126), 1-47; discussion 49-63.

17 Semmler, M., Seitz, J., Erbe, F., Mayer, P., Heyder, J., Oberdorster, G. und Kreyling, W. G., 2004, Long-term clearance kinetics of inhaled ultrafine insoluble iridium particles from the rat lung, including transient translocation into secondary organs, Inhal Toxicol 16(6-7), 453-9.

18 Elder, A. und Oberdorster, G., 2006, Translocation and effects of ultrafine particles outside of the lung, Clin Occup Environ Med 5(4),

19 Elder, A., Gelein, R., Silva, V., Feikert, T., Opanashuk, L., Carter, J., Potter, R., Maynard, A., Ito, Y., Finkelstein, J. und Oberdorster, G., 2006 Translocation of inhaled ultrafine manganese oxide particles to the central nervous system, Environ Health Perspect 114(8), 1172-8.

20 Elder, A. C., Gelein, R., Finkelstein, J. N., Cox, C. und Oberdorster, G., 2000, Pulmonary inflammatory response to inhaled ultrafine particles is modified by age, ozone exposure, and bacterial toxin, Inhal Toxicol 12 Suppl 4, 227-46.

21 Homma, S., Miyamoto, A., Sakamoto, S., Kishi, K., Motoi, N. und Yoshimura, K., 2005, Pulmonary fibrosis in an individual occupationally exposed to inhaled indium-tin oxide, Eur Respir J 25(1), 200-4.

22 Kotter, J. M. und Zieger, G., 1992, Sarcoid granulomatosis after many years of exposure to zirconium, "zirconium lung ", Pathologe 13(2), 104-9.

23 Poland, C.A., Duffin, R., Kinloch, I., Maynard, A., Wallace, W.A.H., Seaton, A., Stone, V., Brown, S., William, M., Donaldson, K. 2008, Carbon nanotubes introduced into the abdominal cavity of mice show asbestos-like pathogenicity in a pilot study, Nature Nanotechnology, Published online: 20 May 2008.

24 Roller, M. und Pott, F., 2006, Lung tumor risk estimates from rat studies with not specifically toxic granular dusts, Ann N Y Acad Sci 1076, 266-80.

25 Geiser, M., Rothen-Rutishauser, B., Kapp, N., Schurch, S., Kreyling, W., Schulz, H., Semmler, M., Im Hof, V., Heyder, J. und Gehr, P. 2005, Ultrafine particles cross cellular membranes by nonphagocytic mechanisms in lungs and in cultured cells, Environ Health Perspect 113(11), 1555-60.

26 Stone, V., Johnston, H. und Clift, M. J., 2007, Air pollution, ultrafine and nanoparticle toxicology: cellular and molecular interactions, IEEE Trans Nanobioscience 6(4), 33 1-40.

27 Oberdorster, G., Sharp, Z., Atudorei, V., Elder, A., Gelein, R., Lunts, A., Kreyling, W. und Cox, C., 2002, Extrapulmonary translocation of ultrafine carbon particles following whole-body inhalation exposure of rats, J Toxicol Environ Health A 65(20), 1531-43.

28 Nemmar, A., Vanbilloen, H., Hoylaerts, M. F., Hoet, P. H., Verbruggen, A. und Nemery, B., 2001, Passage of intratracheally instilled ultrafine particles from the lung into the systemic circulation in hamster, Am J Respir Crit Care Med 164(9), 1665-8.

Medieninhaber: Österreichische Akademie der Wissenschaften; Juristische Person öffentlichen Rechts (BGBI 569/1921 idF BGBI I 130/2003); Dr. Ignaz Seipel-Platz 2, A-1010 Wien

Herausgeber: Institut für Technikfolgen-Abschätzung (ITA); Strohgasse 45/5, A-1030 Wien;

Erscheinungsweise: Die NanoTrust-Dossiers erscheinen unregelmäßig und dienen der Veröffentlichung der Forschungsergebnisse des Instituts für Technikfolgen-Abschätzung im Rahmen des Projekłs NanoTrust. Die Berichte werden ausschließlich über das Internetportal „epub.oeaw” der Öffentlichkeit zur Verfügung

NanoTrust-Dossier Nr. 003, Mai 2008: epub.oeaw.ac.at/ita/nanotrust-dossiers/dossier003.pdf 